\title{
Stability of Reconstituted Amoxicillin and Potassium Clavulanate Oral Suspensions Marketed in the Democratic Republic of the Congo
}

\author{
Mankulu Kakumba Jocelyn*, Mavanga Mabaya Timothy, Bayebila Menanzambi Tresor, \\ Tshienda Katende Milka, Mbenza Puati Adelard, Mbinze Kindenge Jeremi, \\ Kimbeni Malongo Tresor
}

Department of Pharmaceutics and Drugs Analysis, Laboratory of Quality Control and Drugs Analysis and Diets Survey (LACOMEDA), Faculty of Pharmaceutical Sciences, University of Kinshasa, Kinshasa, Democratic Republic of the Congo

\author{
Email address: \\ jocelyn.mankulu@unikin.ac.cd (M. K. Jocelyn) \\ ${ }^{*}$ Corresponding author
}

\section{To cite this article:}

Mankulu Kakumba Jocelyn, Mavanga Mabaya Timothy, Bayebila Menanzambi Tresor, Tshienda Katende Milka, Mbenza Puati Adelard, Mbinze Kindenge Jeremi, Kimbeni Malongo Tresor. Stability of Reconstituted Amoxicillin and Potassium Clavulanate Oral Suspensions Marketed in the Democratic Republic of the Congo. International Journal of Pharmacy and Chemistry. Vol. 7, No. 1, 2021 , pp. 5-12. doi: $10.11648 /$ j.ijpc.20210701.12

Received: December 14, 2020; Accepted: December 22, 2020; Published: February 23, 2021

\begin{abstract}
Amoxicillin and Clavulanate potassium oral suspensions are one of antibiotics combination mainly available as dry powders for reconstitution. Made of a $\beta$-lactam molecule with a $\beta$-lactamase inhibitor, this combination existence can be justified by the potency of Clavulanate potassium which protects Amoxicillin against bacteria producing $\beta$-lactamases. Once reconstituted, suspension in single dose bag need to be taken by patient on daily basis following the medical prescription and the one in bottle to be taken according to the medical prescription and the remaining part (in the same container) kept refrigerated in order to get the optimal benefit from the drug. This study investigated the stability of Amoxicillin and Clavulanate potassium suspension both in bags (sachets) for single dose per daily-usage and in bottles for multiple dose usage. The last were reconstituted using distilled water and stored in refrigerator and the previous were instantly reconstituted before measurement. The stability evaluation was conducted on three brands (Six samples totally, two per brand including bottle and sachet for each brand) and for duration of seven days using a validated HPLC method. The evaluation was based on the measurement of $\mathrm{pH}$ values, color observation of reconstituted suspensions stored under refrigerator during 7 days and the active compounds concentrations determination. After analysis, no change was observed in $\mathrm{pH}$ values and a small change of color for multiple dose presentations stored in refrigerator. And the concentrations of suspensions evaluated in day 1 and day 7 showed over $90 \%$ up to seventh day under same storage conditions for both of the Amoxicillin and Clavulanate potassium pharmaceutical presentations. As the study aimed to assess the stability of the suspension in single-dose from bag presentation and in multiple-dose from suspension in bottle, we observed that the reconstituted suspension in distilled water of Amoxicillin + Clavulanate potassium stored in refrigerator is stable for seven days of use, independently from their pharmaceutical presentations either for oral suspensions (powders) in bottles or those in bags for single use after reconstitution.
\end{abstract}

Keywords: Stability, Quality Control (QC), Amoxicillin, Potassium Clavulanate

\section{Introduction}

\subsection{Stability of the Pharmaceutical Product}

The effectiveness of a formulation can be assessed only through its stability evaluation. The stability studies of a pharmaceutical preparation are a routine activity performed in different stages of its development. In all the cases, various conditions of temperature and/or humidity can be used to determine what kind of degradation products may result based on the storage duration [1].

As matter of fact, we can understand that the minor target 
of drug stability is to find out the quality assurance that the product will absolutely remain at a desirable level of fitness throughout its validity along the storage in pharmacy or the treatment regimen [2]. The stability of a pharmaceutical product can be defined as <the capability of its particular formulation (dosage form or drugs product) in a specific container-enclosure system to remain within its physical, chemical, microbiological, therapeutic and toxicological specification throughout its shelf life $>$ [1].

Based on above statement, the first reason of stability testing is to create a confidence to the patient who is suffering from disease that we are taking care of his health to get recovered. Secondly, the reason for stability testing depends on the need of the manufacturer to protect his brand name according to its evidenced and proved effectiveness. The stability tests are commonly used in industry during the development of the drug formulation to choose appropriates recipients, to select the best storage conditions, to determine the claimed shelf life of the product for confirmation of registration, and finally to verify that the formulation method has not changed and can not influence the final product stability [3, 4].

The stability studies of a pharmaceutical product include the evaluation of environmental conditions of storage such as the temperature, light, air, humidity and the type of the packaging. Pharmacopoeial articles must be labeled about their requisite and specific storage conditions as the expiration date of an article depends on certain conditions [5]. It is also important to mention that the stability of a product can be affected because of the duration of storage even under appropriate conditions. In pharmaceutical industry, this alteration is often observed with reconstituted suspension including also Oral powder for reconstituted suspension. Those alterations are various and can be related to the suspension color, the suspension rheology and can finally affect the product dosage in terms of reducing the quantity of active ingredients due to the degradation in the reconstitution medium along the storage moment.

\subsection{Antibiotics for Oral Suspension}

The suspensions are pharmaceutical forms known as systems having two phases; one is the solid phase and disperses itself in the other which is a liquid phase. That formulation attributes make possible the incorporation of low-solubility drugs, of drugs that quickly degrade in water solution, and of excipient modifying the antibiotic's unpleasant taste, added to render such formulations more acceptable. The suspensions are to present redispersion after shaking, particles' constant size, homogeneous aspect, and microbial resistance, since formulations with inappropriate pharmacotechnique can modify the drug's pharmacokinetics and pharmacodynamics (Pharmaceutical Codex, 1994) [6].

Antibiotics for Oral suspension are mainly available as dry powders for reconstitution in an appropriate solvent. Bulk antibiotic formulated for reconstituted suspensions are to be stored in refrigerator for getting the optimal benefit from the drug [1]. We can mention in this stage, antibiotics are presented in multi-dose recipient and single-dose package depending on the type of patient (age, storage condition, price, etc.); this observation led us to study comparatively the stability of Amoxicillin + Potassium Clavulanate presented in multi-dose bottle versus the same product in a single dose package under same conditions, to evaluate the gap of concentration after 7 days of studies for each presentation after reconstitution.

\subsection{Amoxicillin and Potassium Clavulanate Suspension}

Amoxicillin is a $\beta$-lactam antibiotic, which is the standard treatment for respiratory and urinary tract infections. Since it is inactivated by penicillinases this drug is often used in combination with $\beta$-lactamase inhibitors [8].

In 1981, the clavulanic acid and amoxicillin combination was marketed for the first time in the U.K and became the basic antibiotherapy in several bacterial infections [9, 10]. Amoxicillin and potassium Clavulanate oral suspension contain the equivalent of not less than $90 \%$ and not more than $120 \%$ of the labeled amount of amoxicillin and not less than $90 \%$ and not more than $120 \%$ of the labeled quantity of clavulanic acid [11]. Nowadays, the Amoxicillin and potassium Clavulanate oral suspension is available for adults and contains the equivalent quantity of Amoxicillin and Clavulanic acid for adult posology in 1:8 proportion and presented in sachet for single dose after reconstitution.

Let mention in this stage that the Adult dosage of Amoxicillin and Potassium Clavulanate is available in the market in sachet for single dose usage, containing an appropriate quantity of the suspension powder to be used following the treatment regimen and the paediatric dosage of this combination which is available in multi-dose bottle containing the powder of the pharmaceutical product. However, this kind of container can induce many problems during storage after the first opening operation. Those problems include the active ingredient decomposition (under heat, variable humidity, light) and can also result from the treatment duration (Sabot et al., 2001). Particularly, the Amoxicillin is subjected to the fast degradation and can lead the rate lowering of active ingredient and several degradation products [12].

Changes in the chemical, physical and microbiological properties of the medicine can affect its therapeutic potency and increase the toxicity [13]. The decomposition of active compounds in dosage forms may result from many parameters but it is important to find better conditions to keep the product at a desirable level of concentration during its existence in store (in pharmacy or treatment regimen) [14].

This study aimed to assess the Amoxicillin and Potassium Clavulanate stability in multi-dose suspension in bottle (mostly used for children cure) versus the Amoxicillin and Potassium Clavulanate in single dose sachet (commonly used for adult) under the same storage conditions; means all powders in bottle and sachets were reconstituted using distilled water and kept in the refrigerator between $2^{\circ} \mathrm{C}$ and $8^{\circ} \mathrm{C}$ during the 7 days of the study to ensure stability of active ingredients during the regimen, by quantifying the active ingredients (Amoxicillin and Potassium Clavulanate) 
in day 1 and day 7 for each sample subjected to this study.

Analytical methods for Amoxicillin and Clavulanic acid include microbiological assay, ultraviolet spectrometry, High performance liquid chromatographic (HPLC) methods, etc. Early HPLC methods used to submit Amoxicillin and Clavulanic acid to the pretreatment then later the reversedphase Chromatography with ultraviolet (UV) or amperometric detection have been developed [15].

In this study, we used a simple, fast and sensitive analytical method for the simultaneous determination of Amoxicillin and Potassium clavulanate in pharmaceutical dosage (reconstituted powder) using a reversed-phase HPLC with UV detection.

\section{Materials and Methods}

\subsection{Materials and Reagents}

Methanol (HPLC Grade) is provided by Merck (Darmstadt, Germany), Water (Ultra Pure Grade) from Kim Pharma (Kinshasa, D. R. Congo) produced by Milli-Q Plus 185 Apparatus (Billerica, Massachussets-MA, USA), Amoxicillin and Clavulanic acid from Merck (Darmstadt, Germany). All branded samples used for the assay were purchased from pharmacies in Kinshasa (The Democratic Republic of the Congo).

\subsection{Instrument and Method}

Analytical assays were conducted following the reversedphase method using the high performance liquid chromatographic apparatus branded VWR Hitachi coupled to the UV-DAD 5430 (Anvers, Belgium) detector. Chromaster VWR Hitachi software was used to control the HPL system together with a DellTM computer (Hangzhou, China) to record the signal and for the chromatogram interpretation.

We have also used a GRAM FV-220C electronic balance (IPESAGE S. A. S., France), the XBridget C18, 250x4,6mm, $5 \mu \mathrm{m}$ column from Waters (Massachussets-MA, USA) and IKA ${ }^{\circledR}$ C-MAG MS4 (Grosseron S. A. S., France).

\subsection{Chromatographic Conditions}

The separation process was carried under the following condition:

Table 1. Chromatographic conditions.

\begin{tabular}{llll}
\hline Column & XBridget C18, 250x4,6mm, 5 $\boldsymbol{\mu m}$ \\
\hline Mobile phase A & Methanol & & \\
\hline Mobile phase B & Water & & \\
\hline Gradient & Time (minute) & A (\%) & B (\%) \\
\hline & 0 & 5 & 95 \\
& 15 & 95 & 5 \\
& 16 & 5 & 95 \\
& 25 & 5 & 95 \\
Flow rate & $0,9 \mathrm{ml} / \mathrm{min}$ & & \\
UV detection & $\lambda=220 \mathrm{~nm}$ & & \\
Injection volume & $10 \mu 1$ & & \\
Column temperature & $30^{\circ} \mathrm{C}$ & & \\
Concentration & $100 \mu \mathrm{g} / \mathrm{ml}$ & & \\
\hline
\end{tabular}

\subsection{Sample Preparation}

Samples were made of three branded pharmaceutical products grouped according to their dosage and presentation. There were 3 branded products presented as powder for oral suspension in multi-dose bottles and 3 others of the same formulation presented in sachets for single-dose. Each presentation having a different batch number and expiration date. All the samples we used for the assay were under their valid period as shown in the table below.

Table 2. Sample per container and validity period.

\begin{tabular}{|c|c|c|c|c|c|}
\hline Brand code & Batch number & Origin & Dosage (amoxicillin/Pot. Clavul) & Container & Expiration date \\
\hline $\mathrm{AC} 1$ & HZ6874 & England & $100 \mathrm{mg} / 12,5 \mathrm{mg}$ & Bottle, $60 \mathrm{ml}$ & June 2021 \\
\hline $\mathrm{AC} 2$ & 9479 & France & $100 \mathrm{mg} / 12,5 \mathrm{mg}$ & Bottle, $60 \mathrm{ml}$ & December 2020 \\
\hline $\mathrm{AC} 3$ & 9972 & Morocco & $100 \mathrm{mg} / 12,5 \mathrm{mg}$ & Bottle, $60 \mathrm{ml}$ & January 2021 \\
\hline $\mathrm{AC} 4$ & 2797 & England & $1 \mathrm{~g} / 125 \mathrm{mg}$ & Sachet & April 2021 \\
\hline AC5 & JF7353 & France & $1 \mathrm{~g} / 125 \mathrm{mg}$ & Sachet & September 2020 \\
\hline AC6 & HK6E & Morocco & $1 \mathrm{~g} / 125 \mathrm{mg}$ & Sachet & December 2020 \\
\hline
\end{tabular}

Three samples ( 3 bottles) from each brand made of Amoxicillin and Potassium Clavulanate were reconstituted with appropriate distilled water volume and stored in the refrigerator between $2^{\circ} \mathrm{C}$ and $8^{\circ} \mathrm{C}$ during seven days in accordance to the treatment regimen and three others (made of 3 sachets) were every day reconstituted before analysis as required for its use during the treatment.

\subsection{Standard Preparation}

The standard of Amoxicillin and Potassium Clavulanate were obtained from the US Pharmacopoeia and the Pharmaceutical laboratory of Kinshasa (the national accredited laboratory in quality control) respectively. Prepared in the same conditions with analytical samples, this standard was used as the reference in our study.

\subsection{Statistical Analysis}

All the collected data were treated in Microsoft office excel 2013 and the mean rates of Amoxicillin concentration in different samples were compared using the Student test (the $\mathrm{T}$ test). This test was used within a sill significance of 5\% to express the means comparison of samples under same conservation conditions.

\section{Results and Discussion}

Before proceeding to analysis, three brands contained in the bottles were reconstituted using the distilled water 
volume equal to $60 \mathrm{ml}$ and the three others contained in the sachets for single were also reconstituted with $10 \mathrm{ml}$ of distilled water.

The stability evaluation of the analytical samples was based on three parameters:

$$
\text { 1) } \mathrm{pH}
$$

2) Organoleptic characters (color and taste)

3) Active ingredients concentration

a. $\mathrm{pH}$ evaluation

The evaluation of reconstituted suspensions $\mathrm{pH}$ is presented in the table below both for multidose sample and single-use sample:

Table 3. $p H$ values of Amoxicillin and Clavulanate potassium suspensions.

\begin{tabular}{|c|c|c|c|c|c|c|c|}
\hline \multirow{3}{*}{$\begin{array}{l}\text { Day } \\
\text { Sample code } \\
\text { Storage conditions }\end{array}$} & \multicolumn{3}{|c|}{ Multiple-dose samples } & \multicolumn{3}{|c|}{ Single-dose samples } & \multirow[t]{2}{*}{ USP, XXXI,2012 } \\
\hline & $\mathrm{AC} 1$ & $\mathrm{AC} 2$ & AC3 & AC4 & AC5 & AC6 & \\
\hline & \multicolumn{3}{|c|}{ Refrigerator $\left(2^{\circ} \mathrm{C}-8^{\circ} \mathrm{C}\right)$} & \multicolumn{3}{|c|}{ Instantly reconstituted } & \\
\hline Day 1 & 5 & 5 & 5 & 5,4 & 5,4 & 5,4 & 5 to 7,5 \\
\hline Day 7 & 6 & 6 & 6 & 5,4 & 5,4 & 5,4 & 5 to 7,5 \\
\hline
\end{tabular}

The results above show that after reconstitution of dry powder, suspensions kept the same value of $\mathrm{pH}$ for single dose sachet. This is justified by the fact that, for each sample of sachet we used to reconstitute instantly a pack before measurement and was repeated in day 1 and 7. Differently to the multiple dose containers where, the variation was of one unit $\mathrm{pH}$, the sachets used for every sample of single dose packages were from the same batches and not stored after reconstitution and measurement.

Accordingly to the US Pharmacopeia, we observed that the $\mathrm{pH}$ values were satisfying the norms which require the gap from 5 to 7,5 for oral suspension.

b. The color evaluation

After seven days of storage in refrigerator, the reconstituted suspensions from the three multiple dose containers showed a small change of color from white to yellowish for brands $\mathrm{AC} 1$ and $\mathrm{AC} 3$. Up to the single dose sachets, no change was observed as the suspensions were done instantly before analysis or measurement.

c. Analytical dosage

After reconstitution of suspensions, samples were subjected to the successive dilutions to obtain the final concentration of $10 \mu \mathrm{g} / \mathrm{ml}$ of the solution to be injected in LC (injection volume of $10 \mu \mathrm{l}$ ). The same procedure was repeated on day 1 and day 7 before sample analysis.

Let mention that reference standard also was treated like samples and after the LC running; the following chromatograms were obtained in day 1 and 7 .

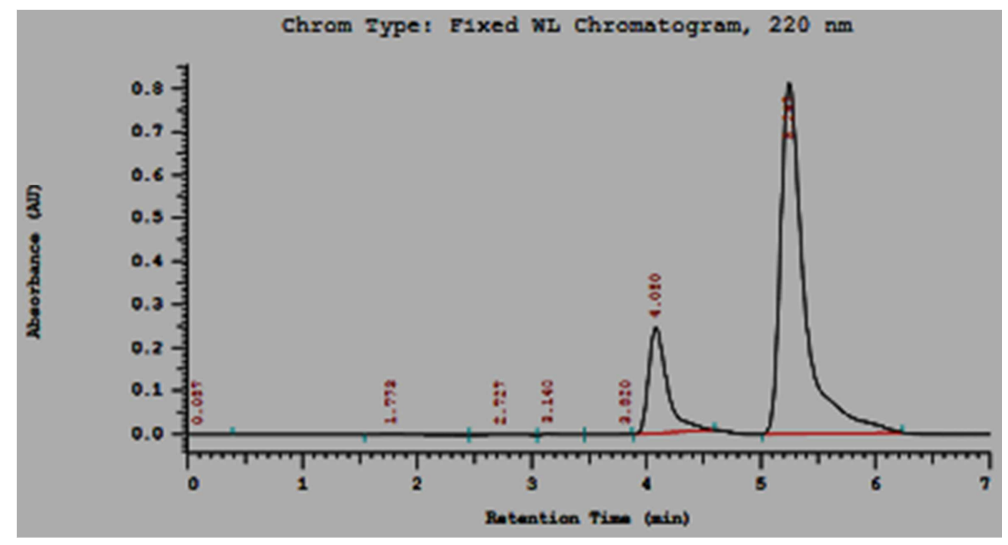

Figure 1. Standard Chromatogram day 1.

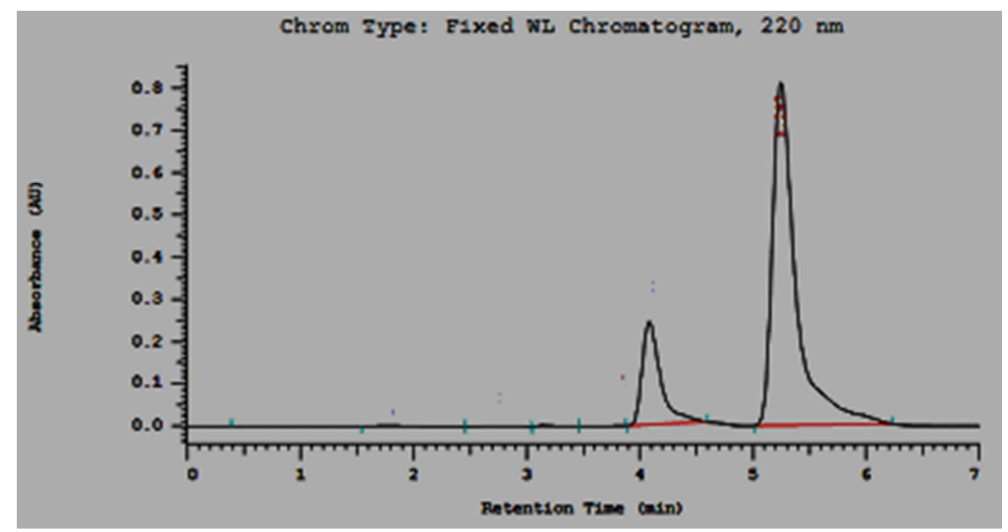

Figure 2. Standard Chromatogram day 7. 
The following chromatographic parameters resulted from the standard analysis during two days (Day 1 and 7) as shown in table 3 .

Table 4. Chromatographic parameters obtained from the standard preparations.

\begin{tabular}{llll}
\hline Day $\mathbf{N}^{\circ}$ & Compounds & Retention time (Minutes) & Area under curve \\
\hline \multirow{2}{*}{1} & Potassium Clavulanate & 4,090 & 1361120 \\
& Amoxicillin & 5,021 & 6011250 \\
7 & Potassium Clavulanate & 4,066 & 1300101 \\
& Amoxicillin & 5,071 & 5861721 \\
\hline
\end{tabular}

After standard analysis, samples were subjected to the same process following the same analytical protocol. This was done in day 1 and day 7 for every sample and obtained the following Chromatogram per sample on daily basis.

$1^{\circ}$. Day 1
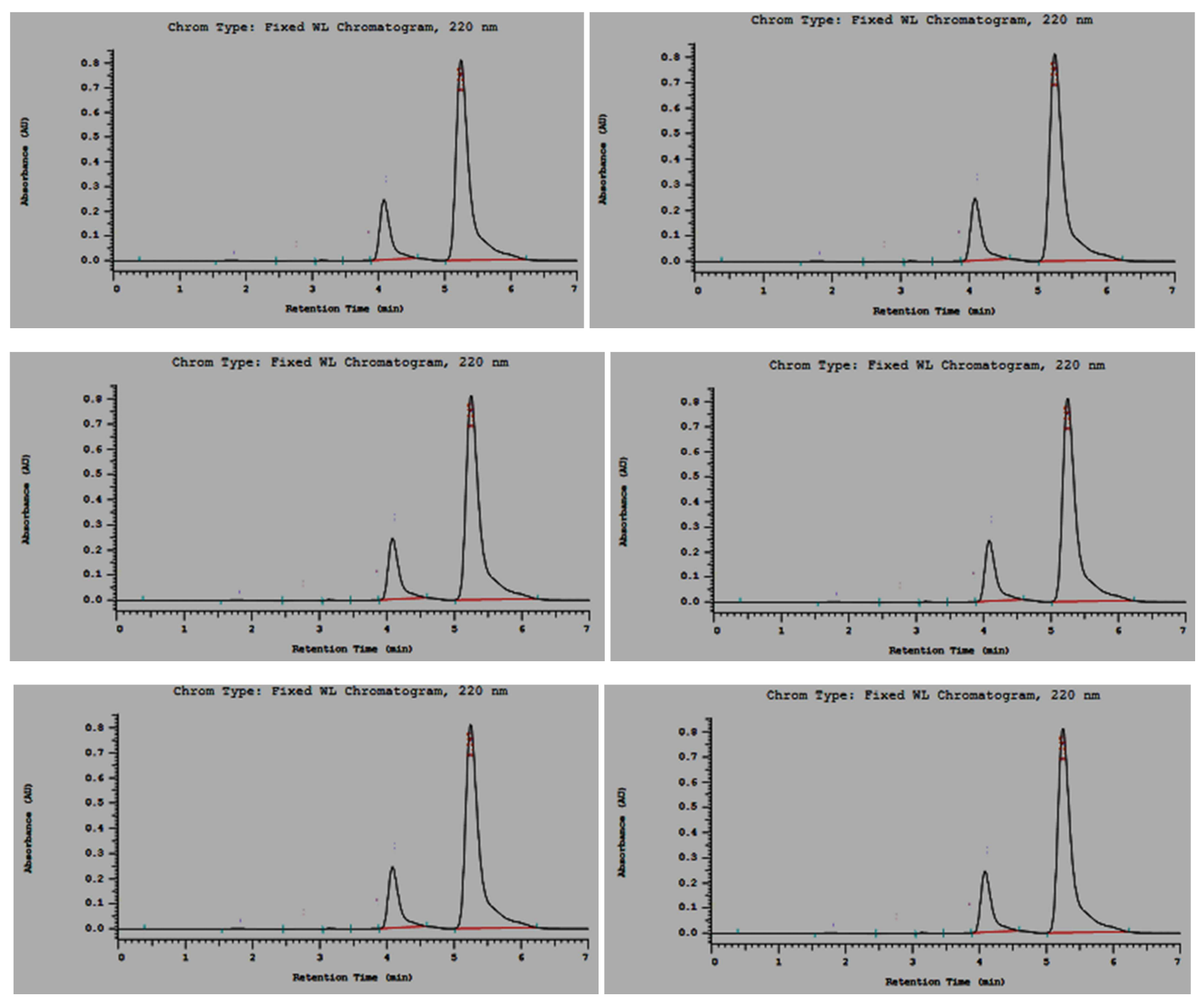

Figure 3. Samples chromatogram day 1.

$2^{\circ}$. Day $7:$ 

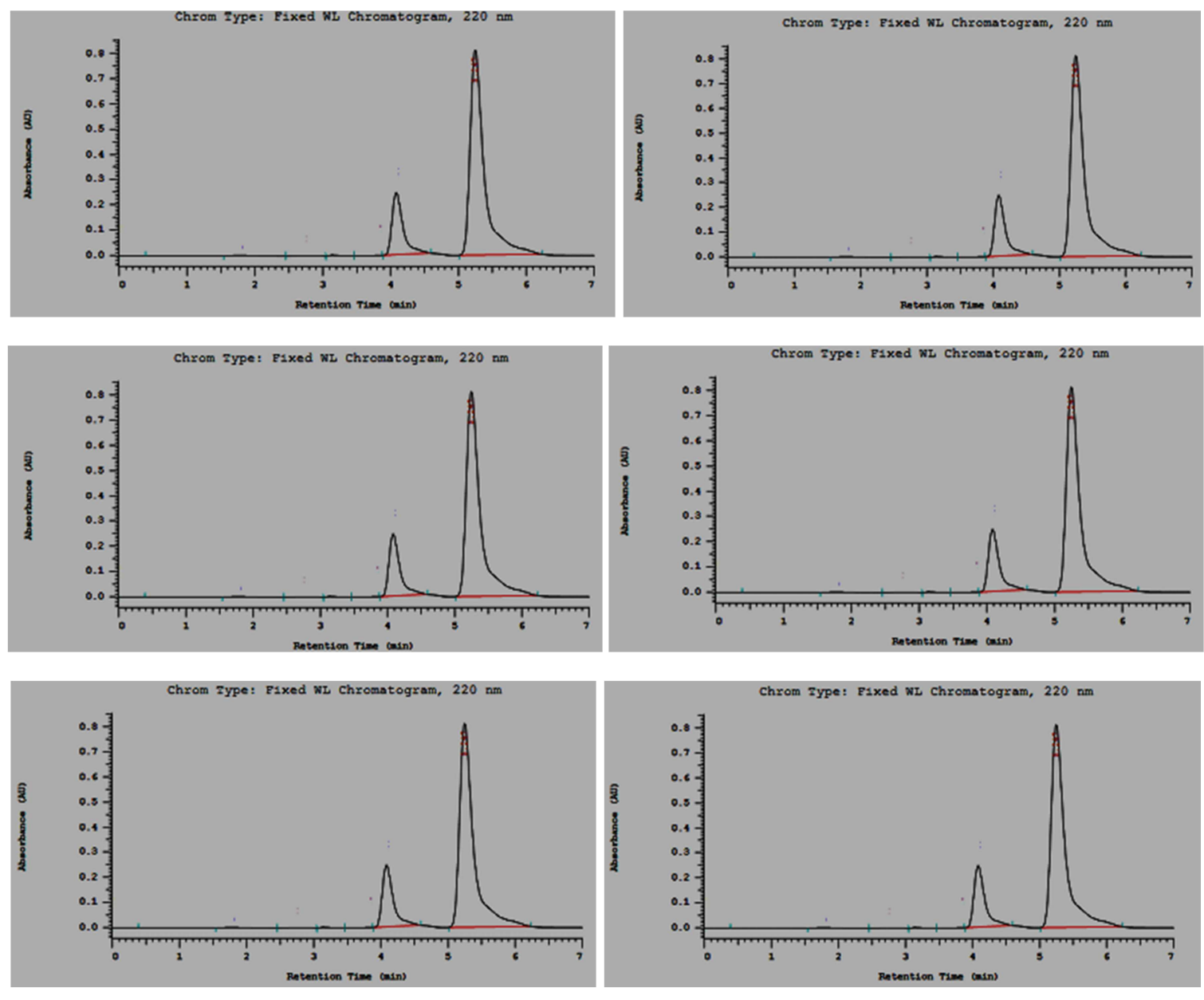

Figure 4. Samples chromatogram day 7.

Bellow these graphs (Chromatograms), you can find Chromatographic parameters and analytical results (Table 5) as obtained with each sample per day in terms of concentration Amoxicillin and Potassium Clavulanate.

Table 5. Chromatographic parameters and analytical results per sample.

\begin{tabular}{|c|c|c|c|c|c|}
\hline Sample & Day $\mathbf{N}^{\circ}$ & Compounds & Retention Time (Minutes) & Area under curve & Concentration (\%) \\
\hline \multirow{4}{*}{$\mathrm{AC} 1$} & \multirow{2}{*}{$1^{\text {st }}$} & Potassium Clavulanate & 4,091 & 1367720 & 100,485 \\
\hline & & Amoxicillin & 5,024 & 6022174 & 100,182 \\
\hline & \multirow{2}{*}{$7^{\text {th }}$} & Potassium Clavulanate & 4,011 & 1244910 & 95,755 \\
\hline & & Amoxicillin & 5,072 & 5712874 & 97,461 \\
\hline \multirow{4}{*}{$\mathrm{AC} 2$} & \multirow{2}{*}{$1^{\text {st }}$} & Potassium Clavulanate & 4,088 & 1360257 & 99,937 \\
\hline & & Amoxicillin & 5,022 & 6202547 & 103,182 \\
\hline & \multirow{2}{*}{$7^{\text {th }}$} & Potassium Clavulanate & 4,066 & 1200101 & 92,308 \\
\hline & & Amoxicillin & 5,071 & 5761721 & 98,294 \\
\hline \multirow{4}{*}{ AC3 } & \multirow{2}{*}{$1^{\mathrm{st}}$} & Potassium Clavulanate & 4,089 & 1298560 & 95,404 \\
\hline & & Amoxicillin & 5,017 & 5935471 & 98,739 \\
\hline & \multirow{2}{*}{$7^{\text {th }}$} & Potassium Clavulanate & 4,044 & 1211276 & 93,168 \\
\hline & & Amoxicillin & 5,013 & 5731026 & 97,770 \\
\hline \multirow{4}{*}{$\mathrm{AC} 4$} & \multirow{2}{*}{$1^{\text {st }}$} & Potassium Clavulanate & 4,041 & 1292459 & 94,956 \\
\hline & & Amoxicillin & 5,011 & 5907865 & 98,280 \\
\hline & \multirow[t]{2}{*}{$7^{\text {th }}$} & Potassium Clavulanate & 4,078 & 1210567 & 93,113 \\
\hline & & Amoxicillin & 5,051 & 5804508 & 99,024 \\
\hline \multirow{4}{*}{ AC5 } & \multirow{2}{*}{$1^{\mathrm{st}}$} & Potassium Clavulanate & 4,040 & 1400235 & 107,702 \\
\hline & & Amoxicillin & 5,011 & 6105225 & 104,154 \\
\hline & \multirow{2}{*}{$7^{\text {th }}$} & Potassium Clavulanate & 4,026 & 1400101 & 107,692 \\
\hline & & Amoxicillin & 5,091 & 6000721 & 102,371 \\
\hline \multirow{4}{*}{ AC6 } & \multirow{2}{*}{$1^{\mathrm{st}}$} & Potassium Clavulanate & 4,039 & 1298851 & 95,425 \\
\hline & & Amoxicillin & 5,062 & 6086637 & 101,254 \\
\hline & \multirow{2}{*}{$7^{\text {th }}$} & Potassium Clavulanate & 4,010 & 1200910 & 92,371 \\
\hline & & Amoxicillin & 5,042 & 5919887 & 100,992 \\
\hline
\end{tabular}


The stability of Amoxicillin and Potassium Clavulanate combination suspension in multi-dose bottle versus the same combination in single-dose sachets after reconstitution and storage in same condition during seven days was assessed by determination of compounds rate (concentration) in different samples by in two steps. The first dosage was done the same day of reconstitution (Day 1) before conservation, and it included all the six brands involved in our study. And the last analysis came on seventh day (generally considered as the last day of treatment regimen) to evaluate the same parameter (concentration of active ingredients) then both the day 1 and 7 results were compared to see the gap of rate variation in terms of concentrations due to the environment and storage conditions after their reconstitution.

After data collection and results (Table 5) analysis, we did not observe a significant difference of Amoxicillin and potassium Clavulanate concentrations in samples when we compare the same brand (in one presentation) variation in day 1 and 7. Also, the same thing was observed by comparing the same brand in different presentations (multidose bottle versus single-dose pocket). Despite the fact that the suspension in single-dose sachet was subjected to the long conservation, we have observed that the Amoxicillin and Potassium Clavulanate concentrations in samples remain in the tolerate level in accordance with the specified standard of US Pharmacopoeia [7]. However, stored inhome conditions the situation is not the same as Nwokoye Peace et al. stated in their study on the stability of reconstituted Amoxicillin-Clavulanate potassium under simulated in-home storage conditions. Their results showed that, under in-home storage conditions $\left(27-29^{\circ} \mathrm{C}\right)$, the degradation was extensive by seventh day with Amoxicillin concentration less than $80 \%$ and Clavulanate concentration falling below $70 \%$.

According to our study, the analysis of samples and standard were conducted daily for determination of active compounds concentrations. The statistical analysis by Student test of $T$ given by mean values showed that there was not difference between the result obtained for each sample in day 1 and the result obtained in day 7. And per brand, the Student test compared the means by comparison of results variation between presentations (Bottles and sachets). The study showed also the similarity between results which were not different for suspensions in multi-dose container (Bottles) and those in single-dose package (Sachets).

\section{Conclusion}

We evaluated the stability of the reconstituted suspension of Amoxicillin and Potassium Clavulanate in multi-dose bottle versus the same combination in single-dose sachets during 7 days as required for the treatment regimen.

The study was conducted using a simple and validated HPLC to separate chemical compounds; based on the determination of Amoxicillin and Potassium Clavulanate concentrations in suspension during the storage period (day 1 and 7), this study used a common and similar analytical protocol. Therefore all samples were reconstituted and stored in refrigerator between $2^{\circ} \mathrm{C}$ and $8^{\circ} \mathrm{C}$ for multiple dose containers (bottles) and those in single-dose packages were reconstituted instantly before measurement. After analysis all the samples gave values above the minimum of the specification in the day 7 after reconstitution.

This study demonstrated that the stability of the reconstituted suspensions based on Amoxicillin and Potassium Clavulanate can be preserved during the period of use either in multi-dose presentation or in single-dose package, after being reconstituted and stored in refrigerator. This is about the concentrations of Amoxicillin and Potassium Clavulanate, which is very important for the therapeutic efficacy during the treatment regimen. The study also demonstrated that there was no change in $\mathrm{pH}$ values during the whole period of storage both for multiple dose presentation and the single dose package. But the experiment showed a small change in color for the multiple dose packages, which does not affect the drug concentration and so, its effectiveness.

\section{Acknowledgements}

To the whole team of the laboratory of quality control and drugs analysis and diets survey (LACOMEDA).

To the local pharmaceutical labs, the pharmaceutical laboratory of Kinshasa (LAPHAKI) and Kim Pharma for supplying us with reagents and solvents.

\section{References}

[1] Ravouru N. and Rajesh K. (2008). Stability evaluation of amoxicillin and potassium Clavulanate tablets USP by accelerated studies, Turk J. Pharm. Sci. (3), 201-214.

[2] Faheem A. A., Gamil Q. O. and Farouk A. A. (2016). Quality and stability of Amoxicillin-Potassium Clavulanate drugs marketed in Yemen: Influence of tropical storage conditions, Journal of Chemical and Pharmaceutical Research, 8 (6), 160166.

[3] Singh S. and Bakshi M. (2000). Guidance on conduct of stress test to determine inherent stability of drugs, Pharm Technol Asia, Special Issue, 24-36.

[4] Carstensen J. T. (2000). Drug stability, Principles and Practices, Marcel Dekker-New York.

[5] John N. A. A., Lawrencia Y. A. and Reimmel K. A. (2014). Stability studies on reconstituted Amoxicillin-Clavulanic Acid oral powder by HPLC Method development and quantification, International Journal of Pharmaceutical Science and Practice, Vol. $3 \mathrm{~N}^{\circ} 1,1-12$.

[6] Blanca E. O. M., Maria R. W. K., Elizabeth W. M. and Paulo C. P. R. (2014). Evaluation of the quality and stability of amoxicillin oral suspension, Journal of Applied Pharmaceutical Science, Vol. 4 (07), 038-040. 
[7] Nwokoye P., Oyetunde O. and Akinleye M. (2012). Stability of reconstituted amoxicillin Clavulanate potassium under simulated in-home storage conditions, Journal of Applied Pharmaceutical Science 02 (01), 28-31.

[8] Lehmann A., Katerere D. R. and Dressman J. (2018). Drug Quality in South Africa, Journal of Pharmaceutical Sciences, $1-26$.

[9] Berry V., Hoover J.; Singley C. and Woodnutt G. (2005). Antimicrob Agents Chemother 49, 908-915.

[10] White A. R., Kaye C., Poupard J.; Pypstra R., Woodnutt G. and Wynne B. (2004), J. AntimicrobChemother, 53, i1-i20.

[11] Banker G. S. (2002), Drug products. Rhodes (Eds) Modern pharmaceutics, Marcel Dekker-New York, 1-21.
[12] N'ghorand T. Y. J. S., Kpaibe S. A. P., Bony N. F., Soko Y. N. and Ake M. (2019). Study of chemical stability of oral suspensions based on amoxicillin, Int. J. Biol. Chem. Sci. 13 (3), 1837-1845.

[13] Ahuja S. (1998). Marcel Dekker-New York, USA.

[14] FDA. (2013), Draft Guidance for industry: Stability testing of new drug substances and products Q1a (R2).

[15] Chaitanya K. A., Chelladurai R., Jeevanantham S., Vignesh R. and Baskaran R. (2012), A novel and high-throughput method for the simultaneous determination of amoxicillin and clavulanic acid in human plasma by liquid chromatography coupled with tandem mass spectrometry, International Journal of Pharmacy and Pharmaceutical Sciences, Vol. 4 Suppl. 3, 648-652. 